\title{
Femtosecond-scale response of GaAs to ultrafast laser pulses
}

\author{
Traian Dumitrică* and Roland E. Allen \\ Department of Physics, Texas A\&M University, College Station, Texas 77843
}

(Received 30 May 2002; published 6 August 2002)

\begin{abstract}
We present nonadiabatic simulations of the coherent response of crystalline GaAs irradiated by intense femtosecond-scale laser pulses. Above a threshold fluence, which corresponds to promotion of about $12 \%$ of the valence electrons into the conduction band, the lattice is destabilized and the electronic band gap collapses to zero. This confirms the nonthermal nature of recently observed structural changes driven by electronic excitations and occurring during the first few hundred femtoseconds.
\end{abstract}

PACS number(s): 71.55.Eq

Recent progress in femtosecond-scale laser pulses has made feasible laboratory experiments at unprecedented powers, magnetic fields, pressures, temperatures, and accelerations. There is great potential for applications, including development of high-frequency lasers and investigation of the properties of plasmas and condensed matter under extreme conditions. At the same time, there is increasing theoretical interest in nonperturbative calculations which include intense laser fields. ${ }^{1}$

In particular, the interaction of femtosecond-scale laser pulses with semiconductor materials has attracted much attention. $^{2-8}$ One would therefore like to understand the fundamental physical processes in this newly accessible regime of short time scales and high intensities.

Experimental observations employing ultrafast optical techniques show that the response of a semiconductor to a femtosecond-scale laser pulse (with a duration of order 100 fs or less) is fundamentally different than the response to a picosecond-scale laser pulse (with a duration of 1 ps or more). Whereas the longer pulses appear to produce ordinary heating of the sample by phonon emission, there is convincing evidence that ultrafast pulses induce a structural transition by directly destabilizing the atomic bonds. For example, Saeta et l. $^{2}$ reported melting of GaAs after $100 \mathrm{fs}$, as evidenced by vanishing of the reflected second-harmonic signal (a signature of a symmetry change in the material). Other important experiments ${ }^{3,4}$ concluded that the response of the dielectric function is dominated by changes in the electronic band structure rather than by the optical susceptibility of the excited free carriers.

Motivated by these experiments, we have performed simulations of the initial stages of the interaction of a laser pulse with a semiconductor, which show that ultrafast disordering can be explained solely through destabilization of the covalent bonds by direct electronic excitation. We employ tight-binding electron-ion dynamics, ${ }^{9}$ a method applicable to general nonadiabatic processes, including the interaction with an intense radiation field. This technique permits simulation of the coupled dynamics of valence electrons and ion cores in a molecule or material. Coupling of atomic orbitals to the external electromagnetic field is also included, so that valence electrons are naturally promoted to the conduction bands as a laser pulse is applied. In the present studies, a 64-atom simulation cell was employed. This is almost an order of magnitude larger than the cells used in previous studies (including the pioneering work of Ref. 8), and the larger number of degrees of freedom provides increased confidence in the reliability of the results. In addition, the present results are more directly comparable to experiment, because they involve the same properties that are monitored in pump-probe measurements: the dielectric function, which is mostly determined by the electronic structure, and the nonlinear susceptibility, which is mostly determined by the symmetry of the system.

Following the initial rapid excitation by the laser pulse, the energy transfer from "hot" photoexcited electrons to the lattice occurs on a slower picosecond time scale, through a variety of carrier-lattice thermalization processes. ${ }^{5}$ With ultrashort pulses, therefore, it is possible to deposit energy in the carrier system on a time scale shorter than the phonon emission time, leading to a nonequilibrium population of "hot" electrons with a still "cold" atomic lattice. Since the electronic configuration is not the ground state, the ion positions are no longer in equilibrium. If a high enough fraction of electrons are promoted from bonding to antibonding states, the crystal becomes unstable, and a structural transition occurs.

Standard molecular dynamics techniques assume the validity of the Born-Oppenheimer approximation. The electrons are required to follow the motion of the nuclei adiabatically, remaining in a ground-state configuration for which the one-electron states are determined by the time-independent Schrödinger equation. The population of the one-electron states is thus assumed to remain invariant during the time evolution of the system. Clearly the Born-Oppenheimer approximation is not relevant to the present problem, when a non-negligible fraction of the valence electrons are promoted to excited states.

Excited-state electron-ion dynamics is a mixed quantumclassical model designed for nonadiabatic processes. Two coupled equations describe the dynamics, ${ }^{9}$

$$
\begin{gathered}
i \hbar \frac{\partial \Psi_{j}}{\partial t}=H(\mathbf{R}, t) \Psi_{j}, \\
M \ddot{\mathbf{R}}=-\sum_{j} \Psi_{j}^{\dagger} \frac{\partial H(\mathbf{R}, t)}{\partial \mathbf{R}} \Psi_{j}-\frac{\partial U_{r e p}}{\partial \mathbf{R}} .
\end{gathered}
$$

Here $H$ represents the usual one-electron Hamiltonian, which is explicitly time dependent when an electromagnetic field is present, and $U_{\text {rep }}$ models the repulsive forces between the ion cores. Equation (2) represents the generalization of the 


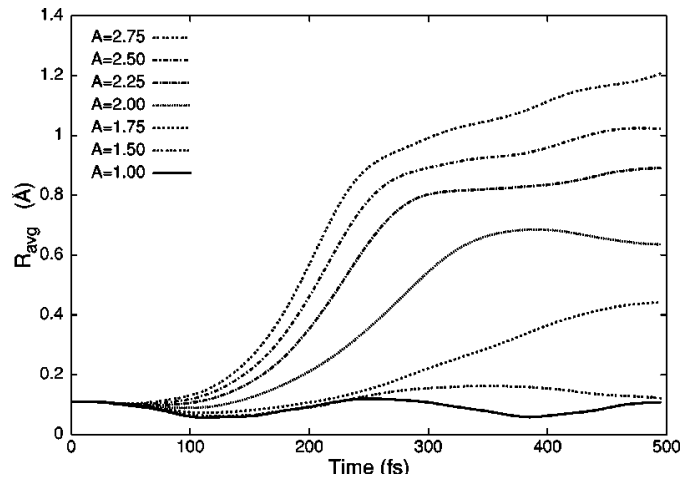

FIG. 1. Average displacement of GaAs atoms from their equilibrium positions for various intensities of the applied laser pulse. In all the simulations reported here, the full-width-at-half-maximum pulse duration is $70 \mathrm{fs}$, and the complete pulse extends from 0 to $140 \mathrm{fs}$. The field intensity is indicated on the upper left, with the amplitude measured in $\mathrm{G} \mathrm{cm}$.

Hellmann-Feynman theorem for the case where $\Psi_{j}$ is no longer an eigenstate of the Hamiltonian. The conjunction of the above two equations leads to a strongly interdependent picture: The electronic states determine the forces on the atoms, while the motion of the atoms (together with the applied electromagnetic field) determines the electronic states.

In a tight-binding representation, the electrons can be coupled to an arbitrarily strong electromagnetic field through a time-dependent Peierls substitution. ${ }^{10,11}$ No additional parameters are required, and the applied field enters simply as a phase in the interatomic matrix elements,

$$
\mathbf{H}_{a b}\left(\mathbf{R}-\mathbf{R}^{\prime}\right)=\mathbf{H}_{a b}^{0}\left(\mathbf{R}-\mathbf{R}^{\prime}\right) \exp \left(-\frac{i e}{\hbar c} \mathbf{A}(t) \cdot\left(\mathbf{R}-\mathbf{R}^{\prime}\right)\right),
$$

where $\mathbf{H}_{a b}^{0}\left(\mathbf{R}-\mathbf{R}^{\prime}\right)$ represents the no-field Hamiltonian matrix element between the atomic orbitals $a$ and $b$, centered on atoms situated at $\mathbf{R}$ and $\mathbf{R}^{\prime}$, respectively. The above phase factor introduced by the vector potential is similar to that in a Bloch state. As a result, the substitution (3) is equivalent to a time-dependent shift in the wave vector, $\mathbf{k}_{A}=\mathbf{k}$ $-(e / \hbar c) \mathbf{A}(t)$, where, as above, $\hbar c / e$ represents the oneelectron flux quantum.

\section{Number of Neighbors}

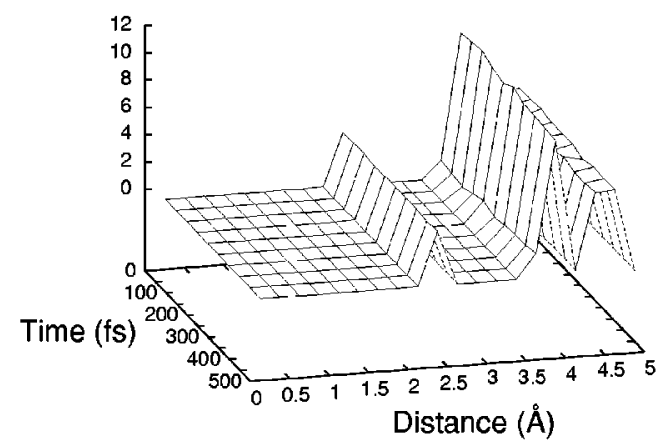

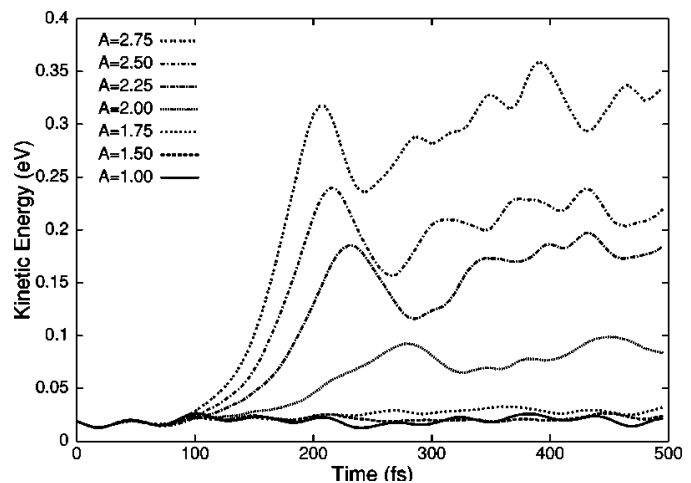

FIG. 2. Kinetic energy of GaAs atoms for various intensities of the applied laser pulse.

To model a laser pulse of total duration $t_{0}$, the vector potential is taken to be $\mathbf{A} \cos \left[\pi\left(t-t_{0} / 2\right) / t_{0}\right] \cos (\omega t)$, where $0 \leqslant t \leqslant t_{0}$. We choose to measure the pulse strength $A$ in gaussian units. A common experimental measure of pulse intensity is the fluence in $\mathrm{kJ} / \mathrm{m}^{2}$. One can relate the vector potential strength $A$ to the fluence by $F=\omega^{2} A^{2} t_{0} / 16 \pi c .{ }^{8}$ Here we employ $\hbar \omega=1.95 \mathrm{eV}$ and $t_{0}=140$ fs. This yields the simple connection $F\left[\mathrm{~kJ} / \mathrm{m}^{2}\right]=0.815(A[\mathrm{G} \mathrm{cm}])^{2}$.

Although a first-principles formulation in the linear combination of atomic orbitals basis is possible, ${ }^{11}$ we find that a tight-binding representation is preferable for the present problem:

(i) The electronic excitations play a central role, so it is important that the excited states be at their proper energies. (These are fitted to experiment in a semiempirical tightbinding model, whereas they are typically too low in the local-density approximation and too high in the Hartree-Fock approximation.)

(ii) To include the oscillatory external field, the time step in the simulations is of order 50 attoseconds, and the system may contain many atoms. As a result, only semiempirical methods are computationally tractable.

The tight-binding model of $\operatorname{Vogl}^{8,12}$ was employed here. Periodic boundary conditions were imposed on the motion of the ions, and simulations were performed in a cubic cell containing 64 atoms. With an appropriate choice for the cutoff function, the model can describe changes in bond length of up to $30 \%$ (so that the initial stages of the excitation are

\section{Number of Neighbors}

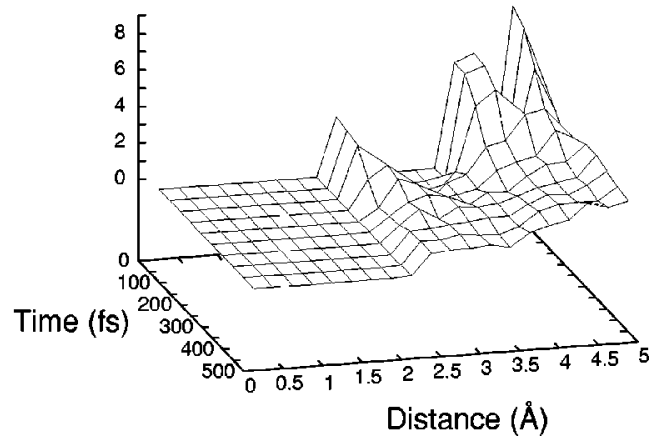

FIG. 3. Time evolution of the pair correlation function for GaAs, at $A=1.5 \mathrm{G} \mathrm{cm}$ (left), and $2.25 \mathrm{G} \mathrm{cm}$ (right). 


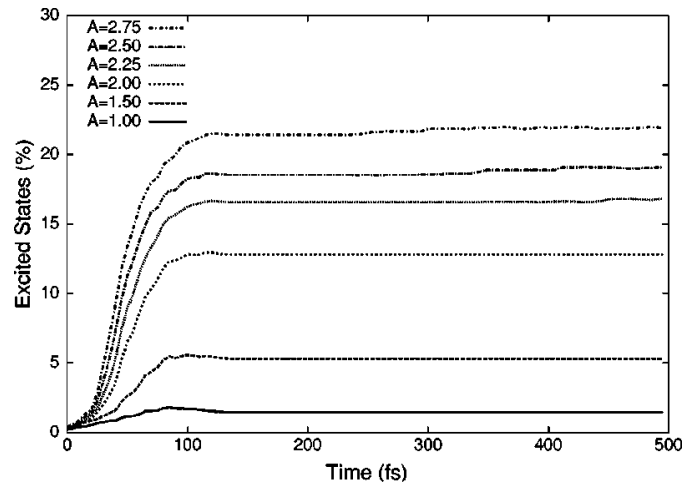

FIG. 4. Occupancy of excited states for pulses of various amplitudes.

reliably described). The second order equation (2) was solved with a velocity Verlet algorithm, which preserves phase space. The first order equation (1) was solved with a Cayley algorithm, which conserves probability ${ }^{11}$ (or more generally preserves the orthonormality of the one-electron wave functions).

We performed simulations at various pulse fluences and monitored the evolution of a set of structural indicators. Together, they should provide a reliable description of the laser melting process. The average atomic displacement and the average kinetic energy per particle (which is equivalent to ionic temperature) are monitored during the whole simulation after each 5 fs time interval. The pair-correlation function, defined as the number of atoms within each particular distance range, is another powerful indicator which reflects very closely the laser-induced changes in the material.

The time evolution of the average atomic displacement and the kinetic energy are shown in Figs. 1 and 2, and Fig. 3 shows the evolution of the average number of neighbors for two representative amplitudes of the external field. In the low intensity regime, up to $A=1.75 \mathrm{G} \mathrm{cm}$, the thermal lattice vibrations and kinetic energy are simply enhanced. As can be seen in Fig. 3, there are no structural changes for $A$ $=1.5 \mathrm{Gcm}$, since the distinction between first, second and third neighbors is preserved. The curve for $A=1.75 \mathrm{G} \mathrm{cm}$ marks the limit of the low fluency region; despite a large average displacement from equilibrium, the changes in kinetic energy are relatively small.

For $A=2.0 \mathrm{G} \mathrm{cm}$ and above, the atoms undergo large displacements from their original positions, and at the same time their kinetic energy increases substantially. The strong diffusive behavior in the $R_{a v g}$ curves is interrupted by a "knee" which appears after 200 fs. This behavior originates from the second-neighbor interaction (as we clearly observed in test runs with different cutoff values for this interaction). The fact that second-neighbor interactions become prominent is a strong signature that the crystal loses its original structure. Figure 3, showing the evolution of the number of neighbors for the case of $A=2.25 \mathrm{G} \mathrm{cm}$, complements this picture: the broadening of the first peak is a strong signature of a change in tetrahedral bonding, whereas the closing up of the valleys between neighbors indicates a disordered phase.

The features of the macroscopic simulations above $A$ $=2.0 \mathrm{G} \mathrm{cm}$ lead us to conclude that the material undergoes a structural transformation of a nonthermal nature. The behavior in these graphs clearly arises from the repulsive forces between atoms when the electrons are promoted to antibonding states, and not from a release of the energy from excited electrons to the lattice.

Although $\Psi_{j}(t)$ can be regarded as the physical state of electron $j$, it is also possible to define eigenvectors $\Phi_{m}(\mathbf{k})$ of the time-dependent Hamiltonian matrix,

$$
\mathbf{H}(t) \Phi_{m}(\mathbf{k})=\varepsilon_{m}(\mathbf{k}) \Phi_{m}(\mathbf{k}) .
$$

The occupancy of the $k$ th state is then given by

$$
n_{k}=\sum_{j}\left|\Psi_{j}^{\dagger} \Phi_{k}\right|^{2}
$$

where $k \leftrightarrow \mathbf{k}, m$.

When an ultrashort laser pulse is applied to a semiconductor, valence electrons are promoted to the conduction bands on a time scale which is short compared to that for atomic motion $(\sim 10-100$ fs versus $\sim 100-1000$ fs). The total occupancy for all the conduction bands is plotted as a function of time in Fig. 4, where it is expressed as a percentage of the total number of valence electrons. One can see that the threshold value of $A=2.0 \mathrm{G} \mathrm{cm}$ corresponds to $12 \%$ promo-
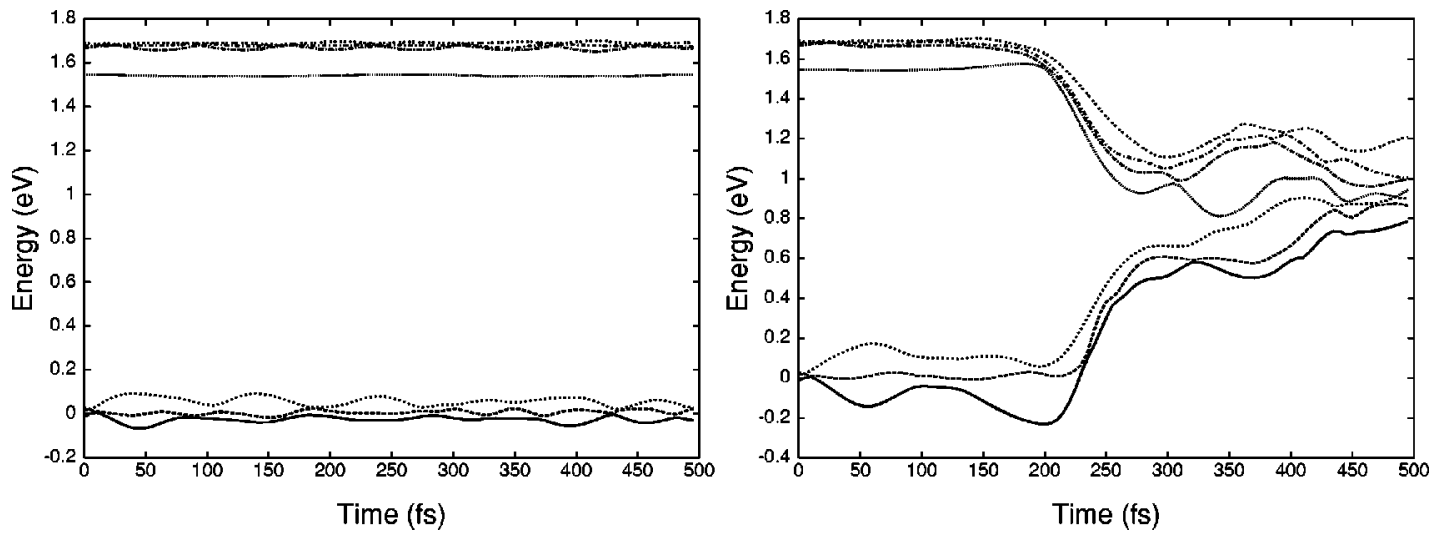

FIG. 5. Electronic energy eigenvalues around the band gap at the $\Gamma$ point as a function of time, for $A=1.5 \mathrm{G} \mathrm{cm}(\mathrm{left})$ and $2.5 \mathrm{G} \mathrm{cm}$ (right). 


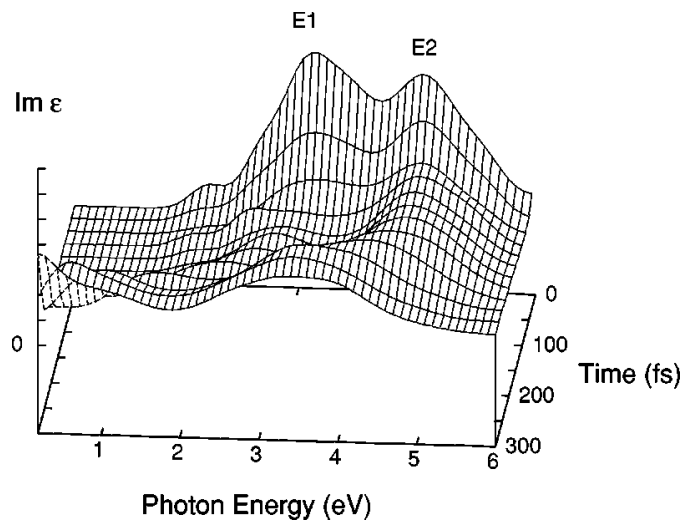

FIG. 6. Imaginary part of the time-dependent dielectric function (in arbitrary units) for an above-threshold intensity corresponding to $A=2.5 \mathrm{G} \mathrm{cm}$.

tion of the valence electrons to conduction-band states. The effect is roughly the same as removing $24 \%$ of the bonds. There are consequently strong repulsive interactions in the initial atomic geometry, which produce massive disruption during the first few hundred femtoseconds.

An amplitude $A=1.0 \mathrm{G} \mathrm{cm}$ corresponds to a fluence of $0.815 \mathrm{~kJ} / \mathrm{m}^{2}$. The threshold for permanent structural change is about $2.0 \mathrm{G} \mathrm{cm}$, or $3.26 \mathrm{~kJ} / \mathrm{m}^{2}$. This is about three times as large as the experimental threshold. ${ }^{4}$ Since the present theory yields a dielectric function roughly half that observed experimentally, ${ }^{10}$ one expects the nonlinear response also to be underestimated.

The eigenvalues around the band gap at the $\Gamma=(0,0,0)$ point are plotted in Fig. 5 as a function of time for two different representative intensities. Notice that the band gap exhibits only thermal oscillations for $A=1.5 \mathrm{Gcm}$, and has completely closed up at $A=2.5 \mathrm{Gcm}$ as a result of large atomic displacements.

Experiments ${ }^{4}$ directly measure ultrafast changes in the GaAs dielectric function over the spectral range from the near-IR to the near-UV. The same quantity $\epsilon(\omega)$ can be calculated in the tight-binding approximation., ${ }^{8,10}$ Figure 6 shows the time evolution of $\operatorname{Im} \epsilon(\omega)$ for the above-threshold case of $A=2.5 \mathrm{Gcm}$, when $18 \%$ of valence electrons are promoted into the conduction band. At $t=0, \operatorname{Im} \epsilon(\omega)$ is characterized by two absorption peaks $E_{1}$ and $E_{2}$. They arise from the regions in the band structure where valence and conduction bands are parallel, leading to a high joint density of states for interband transitions. As time increases the original features are washed out, signaling a loss of the original tetrahedral bonding. Im $\epsilon(\omega)$ also becomes nonzero for photon energies below the original band gap energy of 1.4 $\mathrm{eV}$, and in fact begins to exhibit Drude-like behavior at low energies. This semiconductor-to-metal transition has been observed in other materials, including crystalline $\mathrm{Si}^{6}{ }^{6}$ amorphous $\mathrm{GaAs},{ }^{4}$ and $\mathrm{GeSb}$ films, ${ }^{7}$ indicating common patterns of behavior.

The qualitative agreement with experimental data is yet more detailed:

(i) The transformation starts with a sharp rise in $\operatorname{Im} \epsilon(\omega)$ at the bottom end of the spectral range, around $1.4 \mathrm{eV}$.

(ii) Im $\epsilon(\omega)$ shows a residual interband contribution around $3 \mathrm{eV}$ after the semiconductor-to-metal transition has occurred.

Our simulation shows that this contribution originates from the states in the valence and conduction bands which originally produced the $\mathrm{E}_{2}$ peak in unexcited GaAs, and which are much closer in energy after the band-gap collapse. Therefore bonding-to-antibonding transitions occur even after the long range crystalline order is lost.

In summary, using the method of tight-binding electronion dynamics, we have simulated the interaction of ultrashort laser pulses with GaAs. We provide a detailed microscopic picture for the coupled response of electrons and ions when an ultrashort laser pulse with a full width at half maximum duration of $70 \mathrm{fs}$ and a photon energy $\hbar \omega$ of $1.95 \mathrm{eV}$ excites $1-20 \%$ of the valence electrons. The simulations prove that the GaAs lattice can be disrupted on a subpicosecond time scale by direct electronic excitation, when about $12 \%$ of the valence electrons are promoted to the conduction band. Accompanying the structural transformation there is a band-gap collapse, and $\operatorname{Im} \epsilon(\omega)$ begins to exhibits metallic behavior. The simulations presented here show good agreement with experiment in all important respects, and indicate that destabilization of the covalent bonds by direct electronic excitation is the dominant mechanism for the laser-induced phase transition.

This work was supported by the Robert A. Welch Foundation.
*Present address: Center for Nanoscale Science and Technology, and Department of Mechanical Engineering and Materials Science, Rice University, Houston, Texas 77251-1892.

${ }^{1}$ Atoms, Solids, and Plasmas in Super-Intense Laser Fields, edited by D. Batani et al. (Kluwer Academic, New York, 2001).

${ }^{2}$ P. Saeta et al., Phys. Rev. Lett. 67, 1023 (1991).

${ }^{3}$ L. Huang et al., Phys. Rev. Lett. 80, 185 (1998).

${ }^{4}$ J.P. Callan et al., Chem. Phys. 251, 167 (2000).

${ }^{5}$ E. Mazur, in Spectroscopy and Dynamics of Collective Excitations in Solids, Vol. 356 NATO Advanced Study Institute, Series $B$ : edited by B. Di Bartolo (Plenum, New York, 1997).
${ }^{6}$ K. Sokolowski-Tinten et al., Appl. Phys. A: Mater. Sci. Process. 53, 227 (1991).

${ }^{7}$ J.P. Callan et al., Phys. Rev. Lett. 86, 3650 (2001).

${ }^{8}$ J.S. Graves and R.E. Allen, Phys. Rev. B 58, 13627 (1998).

${ }^{9}$ R.E. Allen, Phys. Rev. B 50, 18629 (1994).

${ }^{10}$ M. Graf and P. Vogl, Phys. Rev. B 51, 4940 (1995).

${ }^{11}$ R.E. Allen, T. Dumitrică, and B. Torralva, in Ultrafast Physical Processes in Semiconductors, edited by K.T. Tsen (Academic, New York, 2001).

${ }^{12}$ P. Vogl, H.P. Hjalmarson, and J.D. Dow, J. Phys. Chem. Solids 44, 365 (1983). 